\title{
The science of sex
}

$\mathrm{S}$ ex is a topic that arouses all kinds of curious reactions from blushing and giggling to indignation. Yet, social mores and embarrassment notwithstanding, sex is crucially important. Without it, none of us would be here; in fact, life on Earth would probably not have evolved beyond some simple forms.

Sexual reproduction - the fusion of gametes to recombine the parental genomes into a new genotype-probably emerged around 1.2 billion years ago among primitive eukaryotes. Needless to say, it quickly became a huge success: sex allowed evolution to switch gears from a leisurely walking pace to supersonic speed in the creation of new and increasingly complex forms of life: before sex, genetic diversity resulted only from the random mutation of genes. Sex changed this profoundly.

Sexual reproduction mixes two sets of parental alleles and thereby creates greater diversity within a single generation than is possible with random mutation. Moreover, it means that potentially beneficial mutations are less likely to disappear. Instead, in diploid organisms, it would be stored together with other seemingly useless alleles, within a population's gene pool. These alleles are constantly and randomly shuffled to create new phenotypes better suited to survive and flourish in a changing world. In essence, sex greatly increases the generation of genetic and phenotypic diversity with which selection can work. Yet, while biologists acknowledge the huge importance of sex for evolution, the questions of how and why it emerged in the first place remain unanswered.

Sex is not only important in evolutionary or biological terms it has also had a profound influence on human history, culture and society. Sex inspired Homer's Iliad, Shakespeare's Romeo and Juliet, Boccaccio's The Decameron, and countless other great works. How boring life would be without sex to stir our passions.

Modern societies have increasingly separated the pleasure of the sexual act from its purpose-sexual reproduction-such that sex and sexual identity have become forms of expression, rather than a mere biological function. The invention of contraception has liberated couples to enjoy sexual intercourse without having to deal with the reproductive consequences. It has also had a profound impact on human society, in particular by enabling the sexual revolution that liberated women from fears of unwanted pregnancy. More recently, technologies for assisted reproduction have enabled humans to have babies without sexual intercourse. It is not yet clear whether and how this will affect society, but it could have profound consequences, not least because the techniques are still fairly inefficient.

In this issue, to reflect the importance of sex, we open our new focus series on 'sex and science' that will explore a variety of biological and societal aspects of sex, sexual reproduction and gender. The first article is by Richard Sharpe, who discusses the causes and consequences of decreasing male fertility. In the next issue, Therese Hesketh will explain the strong cultural and societal preference for sons in many societies-with a focus on China and India-and will analyse the social implications of a growing surplus of young men in these countries.

The series aims to cover a range of biological topics including what determines gender and how this process can go wrong, the evolution of sex and its impact on life on Earth, and sexually transmitted diseases (STDs). The fight against HIV/AIDS and the re-emergence of other STDs have highlighted the difficulties of applying public health measures to control a hard-wired evolutionary instinct.

A number of articles will focus on sexual reproduction itself: the neurological mechanisms that make us yearn for sex, and the 'biochemistry of love', which will look at the hormones and neurotransmitters that enable humans to pursue and maintain long and stable relationships beyond the immediate desire for sexual intercourse and reproduction. One article will highlight how biological factors create new health risks for certain couples who seek assisted reproductive therapy (ART), while another contribution will describe current and future advances in reproductive medicine and explore their potential impact on society.

In the English language, sex has a second meaning in addition to the sexual act itself: it also stands for gender, a concept that other articles will explore. Gender has direct consequences for health and health care, as women and men have different health risks and requirements. Moreover, as our brains and our evolution place such a strong emphasis on sex, we will look at how advertisers exploit this biologically determined obsession to sell their products.

We hope that in the months to come, you will enjoy the 'sex and science' series as much as we, the editorial team, have done in preparing it.

\section{Holger Breithaupt}

EMBO reports (2012) 13, 394; doi.10.1038/embor.2012.45 